\title{
Five-loop fermion anomalous dimension for a general gauge group from four-loop massless propagators
}

\author{
P.A. Baikov, ${ }^{a}$ K.G. Chetyrkin ${ }^{b}$ and J.H. Kühn ${ }^{b}$ \\ ${ }^{a}$ Skobeltsyn Institute of Nuclear Physics, Lomonosov Moscow State University, \\ 1(2), Leninskie gory, Moscow 119991, Russian Federation \\ ${ }^{b}$ Institut für Theoretische Teilchenphysik, Karlsruhe Institute of Technology (KIT), \\ Wolfgang-Gaede-Straße 1, 726128 Karlsruhe, Germany \\ E-mail: baikov@theory.sinp.msu.ru, Konstantin.Chetyrkin@kit.edu, \\ johann. kuehn@kit.edu
}

ABstract: We extend the $\mathcal{O}\left(\alpha_{s}^{5}\right)$ result of the analytic calculation of the quark mass anomalous dimension in $\mathrm{pQCD}$ [1] to the case of a generic gauge group. We present explicit formulas which express the relevant renormalization constants in terms of fourloop massless propagators. We also use our result to shed new light on the old puzzle of the absence of even zetas in results of perturbative calculations for a class of physical observables.

Keywords: Perturbative QCD, Renormalization Group

ARXIV EPRINT: 1702.01458 


\section{Contents}

1 Introduction 1

2 Technical preliminaries $\quad 2$

3 Explicit formulas for $L$-loop $Z_{2}$ and $Z_{\bar{\psi} \psi}$ via $(L-1)$ p-integrals 3

$\begin{array}{llr}4 & \text { Results } & 6\end{array}$

5 The puzzle of $\zeta_{4}$ and $\zeta_{6} \quad 8$

6 Conclusions 11

\section{Introduction}

The dependence of the quark masses on the renormalization scale is governed by the quark mass anomalous dimension, defined as

$$
\left.\mu^{2} \frac{d}{d \mu^{2}} m\right|_{g_{0}, m_{0}}=m \gamma_{m}\left(a_{s}\right) \equiv-m \sum_{i \geq 0} \gamma_{i} a_{s}^{i+1},
$$

where $a_{s}=\alpha_{s} / \pi=g^{2} /\left(4 \pi^{2}\right), g$ is the renormalized strong coupling constant and $\mu$ is the normalization scale in the customarily used $\overline{\mathrm{MS}}$ renormalization scheme.

Up to and including four-loop level the anomalous dimension is known since 20 years [2-6]. Some time ago this result has been pushed to five-loop order, albeit for the special case of an $\mathrm{SU}(3)$ theory with $n_{f}$ species of quarks $[1,7]$. In the present paper the corresponding result will be given for an arbitrary compact simple Lie group.

The quark mass anomalous dimension has important implications: the Higgs boson decay rate into charm and bottom quarks is proportional to the square of the respective quark mass at the scale of $m_{H}$, which, for given on-shell mass, depends on the aforementioned anomalous dimension (see, e.g., recent discussions in $[1,7-10]$ ). The generalization of the result from $\mathrm{SU}(3)$ to an arbitrary compact simple Lie group, as presented in this paper, gives additional insight into the structure of the result.

This paper is organized as follows: technical preliminaries will be presented in section 2 . The next section 3 briefly discusses master formulas for the two relevant renormalization constants which define quark mass anomalous dimension. The main result, the generalization of the $\mathcal{O}\left(\alpha_{s}^{5}\right)$ expression for $\gamma_{m}$ from $\mathrm{SU}(3)$ to an arbitrary compact simple Lie group will be presented subsequently in section 4, together with the special case of QED. In section 5 we will discuss in some detail the structure of irrational contributions to the result for $\gamma_{m}$. Our short conclusions will be given in section 6 . 


\section{Technical preliminaries}

The quark mass renormalization constant, $Z_{m}$, is defined as the ratio of the bare and renormalized quark masses, viz.

$$
Z_{m}=\frac{m_{0}}{m}=1+\sum_{i, j}^{0<j \leq i}\left(Z_{m}\right)_{i j} \frac{a_{s}^{i}}{\epsilon^{j}} .
$$

Within the $\overline{\mathrm{MS}}$ scheme $[11,12]$ the coefficients $\left(Z_{m}\right)_{i j}$ are just numbers; $\epsilon \equiv 2-D / 2$ and $D$ stands for the space-time dimension. Combining eqs. (1.1), (2.1) and using the RG-invariance of $m_{0}$, one arrives at the following formula for $\gamma_{m}$ :

$$
\gamma_{m}=\sum_{i \geq 0}\left(Z_{m}\right)_{i 1} i a_{s}^{i}
$$

One possibility to obtain $Z_{m}$ is to use the well-known relation (see, e.g. section 2.2 of [13])

$$
Z_{m}=Z_{\bar{\psi} \psi} / Z_{2}
$$

where $Z_{\bar{\psi} \psi}$ is the renormalization constant of the quark mass operator ${ }^{1}$

$$
[\bar{\psi} \psi]=Z_{\bar{\psi} \psi} \bar{\psi} \psi=Z_{\bar{\psi} \psi} / Z_{2} \bar{\psi}_{0} \psi_{0}
$$

and $Z_{2}$ is the quark field renormalization constant

$$
\psi_{0}=Z_{2}^{1 / 2} \psi
$$

The QED Ward identity implies that $Z_{2} \equiv Z_{V}$, with $Z_{V}$ being the renormalization constant of the quark vector current:

$$
\left[\bar{\psi} \gamma_{\alpha} \psi\right]=Z_{V} \bar{\psi} \gamma_{\alpha} \psi=Z_{V} / Z_{2} \overline{\psi_{0}} \gamma_{\alpha} \psi_{0}=\overline{\psi_{0}} \gamma_{\alpha} \psi_{0}
$$

To compute $Z_{2}$ and $Z_{\bar{\psi} \psi}$ one starts from the corresponding bare vertex functions (we assume that the only external momentum, $q$, is flowing in and out through the fermion legs)

$$
\gamma_{\alpha} \Gamma_{B}^{V}\left(a_{s}^{0}, q^{2}\right)=\gamma_{\alpha}\left(1+\delta \Gamma_{B}^{V}\left(a_{s}^{0}, q^{2}\right)\right)
$$

and

$$
\Gamma_{B}^{S}\left(a_{s}^{0}, q^{2}\right)=1+\delta \Gamma_{B}^{S}\left(a_{s}^{0}, q^{2}\right)
$$

Requiring the finiteness of the renormalized versions of both vertex functions

$$
\begin{aligned}
& {\left[\Gamma^{V}\right]\left(a_{s}, q^{2}\right)=Z_{V} \Gamma_{B}^{V}\left(a_{s}^{0}, q^{2}\right)=Z_{V}+Z_{V} \delta \Gamma_{B}^{V}\left(a_{s}^{0}, q^{2}\right),} \\
& {\left[\Gamma^{S}\right]\left(a_{s}, q^{2}\right)=Z_{\bar{\psi} \psi} \Gamma_{B}^{S}\left(a_{s}^{0}, q^{2}\right)=Z_{\bar{\psi} \psi}+Z_{\bar{\psi} \psi} \delta \Gamma_{B}^{S}\left(a_{s}^{0}, q^{2}\right)}
\end{aligned}
$$

\footnotetext{
${ }^{1}$ We use square brackets to refer to completely UV renormalized quantities; for simplicity we also do not write explicitly the $\overline{\mathrm{MS}}$ renormalization scale $\mu$.
} 
we arrive at the following recursive equations for $Z_{V}$ and $Z_{\bar{\psi} \psi}$

$$
\begin{aligned}
Z_{V} & =1-K_{\epsilon}\left\{Z_{2} \delta \Gamma_{B}^{V}\left(a_{s}^{0}, q^{2}\right)\right\}, \\
Z_{\bar{\psi} \psi} & =1-K_{\epsilon}\left\{Z_{\bar{\psi} \psi} \delta \Gamma_{B}^{S}\left(a_{s}^{0}, q^{2}\right)\right\},
\end{aligned}
$$

where $K_{\epsilon}\{f(\epsilon)\}$ is the singular part of the Laurent expansion of $f(\epsilon)$ in $\epsilon$ near $\epsilon=0$. Eqs. (2.3), (2.11), (2.12) directly express $Z_{m}$ through massless propagator-type (that is dependent on one external momentum only) Feynman integrals (FI), denoted as p-integrals below.

There exists currently no direct way to analytically evaluate five-loop p-integrals. However, for a given five-loop p-integral we need to know only its pole part in $\epsilon$ in the limit of $\epsilon \rightarrow 0$. The proper use of this fact can significantly simplify our task. The corresponding method - the so-called Infrared Rearrangement (IRR) — first suggested in [14] and elaborated further in [15-17] — allows to effectively decrease the number of loops to be computed by one. ${ }^{2}$ In its initial version IRR was not really universal; it was not applicable in some (though rather rare) cases of complicated FI's. The problem was solved by elaborating a special technique of combined recursive subtraction of both IR and UV divergences — the $R^{*}$-operation $[18,19]$. Formally, the $R^{*}$-operation is defined as a product

$$
R^{*}=R \tilde{R}=\tilde{R} R
$$

where $R$ stands for the Bogoliubov-Parasiuk R-operation $[20,21]$ and $\tilde{R}$ refers to a kind of IR R-operation which subtracts recursively all IR divergences from a given (Euclidean) FI. A detailed discussion of the $R-, \tilde{R}-$ and $R^{*}$-operations and their interplay can be found in $[22,23]$. The technique of the $R^{*}$-operation succeeds in expressing the UV counterterm of every $L$-loop FI in terms of divergent and finite parts of some $(L-1)$-loop massless propagators.

In our case $L=5$ and, using IRR, one arrives at around $10^{5}$ four-loop p-integrals (all Feynman diagrams have been generated with the use of QGRAF [24]). These were reduced to 28 four-loop master p-integrals, which are known analytically, including their finite parts, from [25, 26] as well as numerically from [27].

We have computed the necessary p-integrals with a special version of reduction ${ }^{3}$ which is based on evaluating sufficiently many terms of the $1 / D$ expansion [32] of the corresponding coefficient functions [33]. The algorithm was implemented in a dedicated FORM [34, 35] program.

\section{Explicit formulas for $L$-loop $Z_{2}$ and $Z_{\bar{\psi} \psi}$ via $(L-1)$ p-integrals}

Let us start from eq. (2.12) by rewriting it as follows

$$
Z_{\bar{\psi} \psi}=1-K_{\epsilon} R^{\prime} \delta \Gamma^{S}\left(a_{s}, q^{2}\right)
$$

\footnotetext{
${ }^{2}$ With the price that the resulting p-integrals with one loop less should be evaluated up to and including their constant part in the small $\epsilon$-expansion.

${ }^{3}$ Note, that very recently there has been significant progress in developing direct reduction algorithms (not using $1 / D$ expansion) [28-31].
} 
where $R^{\prime}$ stands for the "incomplete" $R$-operation which, when applied to a FI, subtracts only all its UV subdivergences not touching the UV divergence of the FI as whole (for a good introductory text about renormalization theory and $R$-operation see [36]).

From a purely formal point of view eq. (3.1) is equivalent to (2.12), however the first relation is significantly more flexible as it does not change its form if, for instance, some (or, even, all) propagators in FI's contributing to $\delta \Gamma^{S}\left(a_{s}, q^{2}\right)$ are made massive. To avoid any confusion: by making a propagator $G_{i}(p)$ massive we mean its multiplication by a factor $\frac{p^{2}}{p^{2}-m_{i}^{2}}$. The factor smoothes the behavior of the corresponding Feynman integrands in the region of small momenta of order $m$. It does not contribute in the only integration region relevant for the UV counterm of a given diagram as whole (modulo power suppressed terms of order $m_{i}^{2} / p_{i}^{2}$ and higher) namely the region where all loop momenta are large. This is an obvious consequence of the following statement [37]: any UV counterterm for any logarithmically divergent FI integral does not depend on momenta and masses.

As a result we can transform eq. (3.1) to

$$
Z_{\bar{\psi} \psi}=1-K_{\epsilon} R^{\prime} \delta \Gamma^{S}\left(a_{s}, \underline{m}, q^{2}\right)
$$

where $\underline{m}=m_{1}, \ldots$. The choice of masses in eq. (3.2) and $q^{2}$ is constrained by only one requirement: there should be no IR divergences in its right hand side. The condition of IR safety can be omitted if one lets the $\tilde{R}$-operation remove all IR divergences from the r.h.s. of (3.2).

To proceed we first ignore any IR divergences and choose the $m$-propagators (that is those which are massive in (3.2)) in such a way that the resulting FI's get simpler for calculation. The necessary IR subtractions (if any) will be dealt with later.

A popular possibility is to make all propagators massive with the help of one and the same mass and nullify $q$. The resulting completely massive vacuum graphs are then computed with one or another version of IBP reduction. ${ }^{4}$ The advantages of this "maximally massive" approach are that (i) no IR singularities may appear, (ii) global (unlike local) gauge symmetry stays untouched and, finally, the global renormalization with the help of Z-factors is not very different from the standard one. There is also a price: to compute $Z_{\bar{\psi} \psi}$ on $L$-loop level one should deal with $L$-loop massive vacuum graphs which are significantly more complicated for calculation than $(L-1)$-loop p-integrals.

We will employ a "minimally massive" way, that is the choice of $q=0$ and $\underline{m}=m, 0,0,0, \ldots$ which means that only one line in every FI contributing to $\Gamma^{S}$ is provided with a mass.

In principle, a particular choice of an m-propagator could be tuned (diagramwise!) in many cases in such a way to avoid any IR singularities ${ }^{5}$ and, thus, to avoid any use of $\tilde{R}$ operation. However, such a tuning essentially prevents any possibility to solve combinatoric of both $R$ - and $\tilde{R}$-operations globally in terms of corresponding Z-factors. This, in turn, forces us either to perform an extremely tedious manual diagram-wise renormalization

\footnotetext{
${ }^{4}$ The method is actively used since long [38-40]. Very recent impressive results obtained with the method can be found in [41, 42].

${ }^{5}$ That was exactly the strategy used in the pioneering calculations within IRR method [15, 17, 43-45].
} 


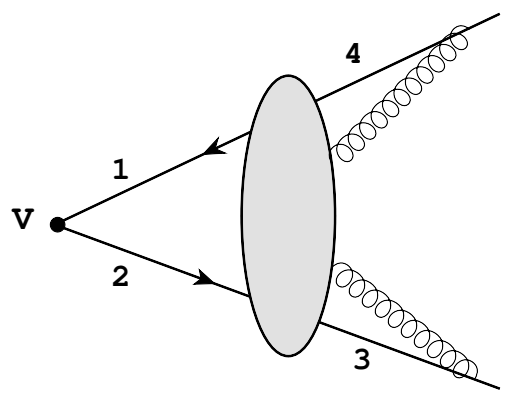

Figure 1. All distinguished fermion propagators available for a generic vector (and scalar) vertex functon.

of UV/IR divergences or to develop highly non-trivial computer algebra routines for its automatization. ${ }^{6}$

Every diagram contributing to $\Gamma^{S}$ has only four internal propagators which could be specified globally without referencing to particular graphs. (This is a necessary requirement allowing us to resolve combinatorics of both UV and IR subtractions in terms of global Zfactors). These are shown on figure 1. Let us choose the fermion line which flows into the scalar current vertex (marked by 1 on figure 1) and make the corresponding propagator massive by multiplying it by $p^{2} /\left(p^{2}-m^{2}\right)$. The result of applying the $R^{\prime}$-operation to the corresponding vertex function $\delta \Gamma^{S}\left(a_{s}, m\right)$ can be expressed in terms of the bare vertex function and relevant Z-factors as

$$
R^{\prime} \delta \Gamma^{S}\left(a_{s}, m\right)=\delta \Gamma_{B}^{S}\left(a_{s}^{0}, m\right)+\delta Z_{\bar{\psi} \psi} \delta \Gamma_{B}^{S}\left(a_{s}^{0}, m=0\right)
$$

where $\delta Z_{\bar{\psi} \psi}=Z_{\bar{\psi} \psi}-1$. The r.h.s. of the above equation is contaminated by IR singularities; they appear not only in the second term but also in the first one. The application of $\tilde{R}$ operation to remove all IR divergent pieces from (3.3) leads to:

$$
\tilde{R} R^{\prime} \delta \Gamma^{S}\left(a_{s}, m\right)=\delta \Gamma_{B}^{S}\left(a_{s}^{0}, m\right)+\left(\delta \Gamma_{B}^{S}\left(a_{s}^{0}, m\right)+\delta Z_{\bar{\psi} \psi}\right) \cdot\left(\frac{1}{Z_{\bar{\psi} \psi}}-1\right)
$$

where we have boxed the IR Z-factor. The considerations for the vector case follow the same pattern with $Z_{\bar{\psi} \psi}$ replaced by $Z_{V}$.

Thus, the final formulas for $Z_{V}$ and $Z_{\bar{\psi} \psi}$ are:

$$
\begin{aligned}
& Z_{V}=1-K_{\epsilon}\left\{\delta \Gamma_{B}^{V}\left(a_{s}^{0}, m\right) \frac{1}{Z_{V}}-\frac{\left(Z_{V}-1\right)^{2}}{Z_{V}}\right\} \\
& Z_{\bar{\psi} \psi}=1-K_{\epsilon}\left\{\delta \Gamma_{B}^{S}\left(a_{s}^{0}, m\right) \frac{1}{Z_{\bar{\psi} \psi}}-\frac{\left(Z_{\bar{\psi} \psi}-1\right)^{2}}{Z_{\bar{\psi} \psi}}\right\} .
\end{aligned}
$$

\footnotetext{
${ }^{6}$ Very recently such routines implementing automatic UV and IR renormalization on the level of separate diagrams have been developed [46].
} 


\section{Results}

Our result for the quark mass anomalous dimension

$$
\gamma_{m}=-\sum_{i}\left(\gamma_{m}\right)_{i} a_{s}^{i+1},
$$

reads (for completeness we also present the lower order terms $[2-4,6]$ )

$$
\begin{aligned}
& \left(\gamma_{m}\right)_{0}=\frac{3}{4} C_{F}, \quad\left(\gamma_{m}\right)_{1}=\frac{1}{4^{2}}\left\{\frac{3}{2} C_{F}^{2}-\frac{10}{3} C_{F} T_{f} n_{f}+\frac{97}{6} C_{F} C_{A}\right\}, \\
& \left(\gamma_{m}\right)_{2}=\frac{1}{4^{3}}\left\{\frac{129}{2} C_{F}^{3}-\left[46-48 \zeta_{3}\right] C_{F}^{2} T_{f} n_{f}-\frac{140}{27} C_{F} T_{f}^{2} n_{f}^{2}-\frac{129}{4} C_{F}^{2} C_{A}\right. \\
& \left.-\left[\frac{556}{27}+48 \zeta_{3}\right] C_{F} T_{f} n_{f} C_{A}+\frac{11413}{108} C_{F} C_{A}^{2}\right\}, \\
& \left(\gamma_{m}\right)_{3}=\frac{1}{4^{4}}\left\{C_{F}^{4}\left[-\frac{1261}{8}-336 \zeta_{3}\right]+C_{F}^{3} T_{f} n_{f}\left[-\frac{280}{3}+552 \zeta_{3}-480 \zeta_{5}\right]\right. \\
& +C_{F}^{2} T_{f}^{2} n_{f}^{2}\left[\frac{304}{27}-160 \zeta_{3}+96 \zeta_{4}\right]+C_{F} T_{f}^{3} n_{f}^{3}\left[-\frac{664}{81}+\frac{128}{9} \zeta_{3}\right] \\
& +C_{F}^{3} C_{A}\left[\frac{15349}{12}+316 \zeta_{3}\right]+C_{F}^{2} C_{A} T_{f} n_{f}\left[-\frac{8819}{27}+368 \zeta_{3}-264 \zeta_{4}+80 \zeta_{5}\right] \\
& +C_{F} T_{f}^{2} n_{f}^{2} C_{A}\left[\frac{1342}{81}+160 \zeta_{3}-96 \zeta_{4}\right]+C_{F}^{2} C_{A}^{2}\left[-\frac{34045}{36}-152 \zeta_{3}+440 \zeta_{5}\right] \\
& +C_{F} T_{f} n_{f} C_{A}^{2}\left[-\frac{65459}{162}-\frac{2684}{3} \zeta_{3}+264 \zeta_{4}+400 \zeta_{5}\right]+C_{F} C_{A}^{3}\left[\frac{70055}{72}+\frac{1418}{9} \zeta_{3}-440 \zeta_{5}\right] \\
& \left.+n_{f} \frac{d_{F}^{a b c d} d_{F}^{a b c d}}{d_{R}}\left[64-480 \zeta_{3}\right]+\frac{d_{F}^{a b c d} d_{A}^{a b c d}}{d_{R}}\left[-32+240 \zeta_{3}\right]\right\}, \\
& \left(\gamma_{m}\right)_{4}=\frac{1}{4^{5}}\left\{C_{F}^{5}\left[\frac{50995}{8}+848 \zeta_{3}+2080 \zeta_{5}\right]\right. \\
& +C_{F}^{4} T_{f} n_{f}\left[-\frac{48797}{36}+6888 \zeta_{3}-672 \zeta_{4}-\frac{37000}{3} \zeta_{5}+6720 \zeta_{7}\right] \\
& +C_{F}^{3} T_{f}^{2} n_{f}^{2}\left[\frac{45253}{54}-\frac{38416}{9} \zeta_{3}+896 \zeta_{3}^{2}+1304 \zeta_{4}+\frac{8000}{3} \zeta_{5}-1600 \zeta_{6}\right] \\
& +C_{F}^{2} T_{f}^{3} n_{f}^{3}\left[\frac{8966}{81}+\frac{352}{3} \zeta_{3}-320 \zeta_{4}+\frac{512}{3} \zeta_{5}\right] \\
& +C_{F}^{4} C_{A}\left[-\frac{2565029}{144}-13060 \zeta_{3}+1848 \zeta_{4}+\frac{29600}{3} \zeta_{5}\right] \\
& +C_{F}^{3} T_{f} n_{f} C_{A}\left[-\frac{406861}{108}-\frac{18013}{9} \zeta_{3}+1760 \zeta_{3}^{2}-2360 \zeta_{4}+5620 \zeta_{5}+4400 \zeta_{6}-3360 \zeta_{7}\right] \\
& +C_{F}^{3} C_{A}^{2}\left[\frac{2625197}{72}+24174 \zeta_{3}-1738 \zeta_{4}-\frac{99140}{3} \zeta_{5}-9240 \zeta_{7}\right] \\
& +C_{F}^{2} T_{f}^{2} n_{f}^{2} C_{A}\left[\frac{375373}{486}+\frac{5996}{9} \zeta_{3}-\frac{6976}{3} \zeta_{3}^{2}+1864 \zeta_{4}-\frac{12448}{9} \zeta_{5}+\frac{800}{3} \zeta_{6}\right] \\
& +C_{F}^{2} T_{f} n_{f} C_{A}^{2}\left[\frac{753557}{1944}+\frac{72194}{9} \zeta_{3}-\frac{5456}{3} \zeta_{3}^{2}-3226 \zeta_{4}-\frac{81464}{9} \zeta_{5}+\frac{2200}{3} \zeta_{6}+560 \zeta_{7}\right]
\end{aligned}
$$




$$
\begin{aligned}
& +C_{F}^{2} C_{A}^{3}\left[-\frac{25256617}{972}-\frac{50642}{3} \zeta_{3}-\frac{1936}{3} \zeta_{3}^{2}+836 \zeta_{4}+\frac{374180}{9} \zeta_{5}-\frac{12100}{3} \zeta_{6}+6160 \zeta_{7}\right] \\
& +C_{F} T_{f}^{4} n_{f}^{4}\left[-\frac{1040}{81}-\frac{1280}{81} \zeta_{3}+\frac{256}{9} \zeta_{4}\right] \\
& +C_{F} T_{f}^{3} n_{f}^{3} C_{A}\left[\frac{18667}{243}+\frac{21472}{81} \zeta_{3}+\frac{2176}{9} \zeta_{4}-\frac{4096}{9} \zeta_{5}\right] \\
& +C_{F} T_{f}^{2} n_{f}^{2} C_{A}^{2}\left[\frac{27418}{243}+\frac{87722}{27} \zeta_{3}+\frac{4288}{3} \zeta_{3}^{2}-\frac{9364}{3} \zeta_{4}-\frac{2944}{3} \zeta_{5}+\frac{4000}{3} \zeta_{6}\right] \\
& +C_{F} T_{f} n_{f} C_{A}^{3}\left[-\frac{4994047}{972}-\frac{959759}{81} \zeta_{3}+\frac{176}{3} \zeta_{3}^{2}+\frac{54925}{9} \zeta_{4}+\frac{139234}{9} \zeta_{5}-\frac{15400}{3} \zeta_{6}-3920 \zeta_{7}\right] \\
& +C_{F} C_{A}^{4}\left[\frac{22663417}{1944}+\frac{418936}{81} \zeta_{3}+\frac{1936}{3} \zeta_{3}^{2}-\frac{15697}{18} \zeta_{4}-\frac{64405}{3} \zeta_{5}+\frac{12100}{3} \zeta_{6}+3080 \zeta_{7}\right] \\
& +C_{F} n_{f} \frac{d_{F}^{a b c d} d_{F}^{a b c d}}{d_{R}}\left[-352-4928 \zeta_{3}+11840 \zeta_{5}\right] \\
& +\frac{C_{F}}{d_{F}^{a b c d} d_{A}^{a b c d}}\left[6768+3840 \zeta_{3}-25440 \zeta_{5}\right] \\
& +T_{R} n_{f}^{2} \frac{d_{F}^{a b c d} d_{F}^{a b c d}}{d_{R}}\left[-\frac{6896}{9}+\frac{7312}{3} \zeta_{3}-768 \zeta_{4}-\frac{2240}{3} \zeta_{5}\right] \\
& +T_{f} n_{f} \frac{d_{F}^{a b c d} d_{A}^{a b c d}}{d_{R}}\left[\frac{3040}{9}-\frac{14920}{3} \zeta_{3}-1408 \zeta_{3}^{2}-144 \zeta_{4}+\frac{3520}{3} \zeta_{5}\right] \\
& +n_{f} C_{A} \frac{d_{F}^{a b c d} d_{F}^{a b c d}}{d_{R}}\left[\frac{25384}{9}-\frac{16720}{3} \zeta_{3}+1408 \zeta_{3}^{2}+2640 \zeta_{4}-\frac{35680}{3} \zeta_{5}\right] \\
& +C_{A} \frac{d_{F}^{a b c d} d_{A}^{a b c d}}{d_{R}}\left[-\frac{76784}{9}-\frac{1208}{3} \zeta_{3}-3872 \zeta_{3}^{2}-1320 \zeta_{4}+23200 \zeta_{5}+1232 \zeta_{7}\right] \\
& \left.+T_{f} \frac{d_{A}^{a b c d} d_{A}^{a b c d}}{d_{R}}\left[358+\frac{4988}{3} \zeta_{3}+3872 \zeta_{3}^{2}+264 \zeta_{4}-\frac{15640}{3} \zeta_{5}-1232 \zeta_{7}\right]\right\} \\
&
\end{aligned}
$$

Here $\zeta$ is the Riemann zeta-function (with $\zeta_{3}=1.2020569 \ldots, \zeta_{4}=1.0823232 \ldots$, $\left.\zeta_{5}=1.0369278 \ldots, \zeta_{6}=1.0173431 \ldots, \zeta_{7}=1.0083493 \ldots\right) . C_{F}$ and $C_{A}$ are the quadratic Casimir operators of the quark $\left[T^{a} T^{a}\right]_{i j}=C_{F} \delta_{i j}$ and the adjoint $\left[C^{a} C^{a}\right]_{b d}=C_{A} \delta_{b d}$, $\left(C^{a}\right)_{b c}=-i f^{a b c}$ representations of the Lie algebra. $n_{f}$ stands for the number of quark flavors, $d_{R}$ is dimension of the quark repesenation of the gauge group and $T_{f}$ refers to the trace normalization $\operatorname{tr}\left(T^{a} T^{b}\right)=T_{f} \delta^{a b}$. The higher order group invariants are defined as contraction between the following (symmetrical) tensors (see [6, 47])

$$
\begin{gathered}
d_{F}^{a b c d}=\frac{1}{6} \operatorname{Tr}\left[T^{a} T^{b} T^{c} T^{d}+T^{a} T^{b} T^{d} T^{c}+T^{a} T^{c} T^{b} T^{d}\right. \\
\left.+T^{a} T^{c} T^{d} T^{b}+T^{a} T^{d} T^{b} T^{c}+T^{a} T^{d} T^{c} T^{b}\right], \\
d_{A}^{a b c d}=\frac{1}{6} \operatorname{Tr}\left[\begin{array}{c}
{ }^{a} C^{b} C^{c} C^{d}+C^{a} C^{b} C^{d} C^{c}+C^{a} C^{c} C^{b} C^{d} \\
+
\end{array}\right. \\
\left.+C^{a} C^{c} C^{d} C^{b}+C^{a} C^{d} C^{b} C^{c}+C^{a} C^{d} C^{c} C^{b}\right] .
\end{gathered}
$$

Note that for the gauge group $\mathrm{SU}(3)$, after setting $C_{F}=4 / 3, C_{A}=3, T_{f}=1 / 2, d_{R}=3$, $d_{F}^{a b c d} d_{F}^{a b c d}=5 / 12, d_{F}^{a b c d} d_{A}^{a b c d}=15 / 2, d_{A}^{a b c d} d_{A}^{a b c d}=135$ we exactly reproduce the SU(3) result for $\gamma_{m}$ given in [1]. 
For the special case of QED, setting $C_{F}=1, T_{f}=1, C_{A}=0, d_{A}^{a b c d}=0, d_{F}^{a b c d} d_{F}^{a b c d}=1$, and $d_{R}=1$ we arrive at

$$
\begin{aligned}
\left(\gamma_{m}^{\mathrm{QED}}\right)_{0}= & \frac{3}{4}, \quad\left(\gamma_{m}^{\mathrm{QED}}\right)_{1}=\frac{3}{32}-\frac{5}{24} n_{f}, \\
\left(\gamma_{m}^{\mathrm{QED}}\right)_{2}= & \frac{1}{4^{3}}\left\{\frac{129}{2}-n_{f}\left[46-48 \zeta_{3}\right]-\frac{140}{27} n_{f}^{2}\right\} \\
\left(\gamma_{m}^{\mathrm{QED}}\right)_{3}= & \frac{1}{4^{4}}\left\{-\left[\frac{1261}{8}+336 \zeta_{3}\right]-n_{f}\left[\frac{88}{3}-72 \zeta_{3}+480 \zeta_{5}\right]+n_{f}^{2}\left[\frac{304}{27}-160 \zeta_{3}+96 \zeta_{4}\right]\right. \\
& \left.-n_{f}^{3}\left[\frac{664}{81}-\frac{128}{9} \zeta_{3}\right]\right\}, \\
\left(\gamma_{m}^{\mathrm{QED}}\right)_{4}= & \frac{1}{4^{5}}\left\{\left[\frac{50995}{8}+848 \zeta_{3}+2080 \zeta_{5}\right]+n_{f}\left[-\frac{61469}{36}+1960 \zeta_{3}-672 \zeta_{4}-\frac{1480}{3} \zeta_{5}+6720 \zeta_{7}\right]\right. \\
& +n_{f}^{2}\left[\frac{3877}{54}-\frac{16480}{9} \zeta_{3}+896 \zeta_{3}^{2}+536 \zeta_{4}+1920 \zeta_{5}-1600 \zeta_{6}\right] \\
& \left.+n_{f}^{3}\left[\frac{8966}{81}+\frac{352}{3} \zeta_{3}-320 \zeta_{4}+\frac{512}{3} \zeta_{5}\right]+n_{f}^{4}\left[-\frac{1040}{81}-\frac{1280}{81} \zeta_{3}+\frac{256}{9} \zeta_{4}\right]\right\} .
\end{aligned}
$$

\section{$5 \quad$ The puzzle of $\zeta_{4}$ and $\zeta_{6}$}

In this section we will consider exclusively "quantities" expressible (read computable) in terms of massless propagators (p-integrals). For any such quantity computed up to some number of loops the result always contains some quite limited number of the irrational constants.

More precisely, if a quantity, say, $P\left(Q^{2}\right)$ can be expressed in terms of p-integrals with the loop number $L$ not exceeding four, then the complete list of the irrational constants reads $\zeta_{3}, \zeta_{4}, \zeta_{5}, \zeta_{6}, \zeta_{3}^{2}, \zeta_{3} \zeta_{4}$ and, finally, $\zeta_{7}$. The reason is obvious, these and only these irrational constants appear in all 4-loop master p-integrals [25]. If we consider $L \leq 3$ then only $\zeta_{3}, \zeta_{4}$ and $\zeta_{5}$ survive [16]. Finally, for $L \leq 2$ there remains only $\zeta_{3}$.

It was already observed long ago that in practice the real pattern of appearance of the irrational constants is somewhat more limited than the one described above.

Indeed, up to now the result of the explicit calculation of any "physical" (in the sense of having no anomalous dimension) quantity $P\left(Q^{2}\right)$ (expressible in terms of p-integrals) has never contained even zetas, that is $\zeta_{4}$ and $\zeta_{6}$. Note that statement is applicable only to Euclidean quantities; it is very well known that terms proportional $\pi^{2}$ are routinely generated during the procedure of analytical continuation to the Minkowskian (negative) values of the momentum transfer $Q^{2}$.

Two most prominent examples are: the Adler function $D\left(Q^{2}\right)$ and the coefficient function $C^{B j p}$ in the Wilson expansion of two vector currents related to the Bjorken sum rules from polarized electron-nucleon scattering (both known up to order $\alpha_{s}^{4}$ [48]).

Some reason behind this remarkable pattern of absence of even zetas from physical quantities $P\left(Q^{2}\right)$ was provided by an analysis of the transcendental structure of the cor- 
responding master p-integrals the level of three [49] and four [25] loops correspondingly. In particular, in [25] the following theorem has been proven (by "any" p-integral in its formulation we mean arbitrary p-integral with the number of loops not exceeding four):

\section{Theorem.}

1. Any p-integral, finite at $\epsilon \rightarrow 0$, does not contain even zetas $\left\{\zeta_{2 n} \mid n \geq 2\right\}$ in the limit of $\epsilon \rightarrow 0$.

2. Any combination of p-integrals, finite at $\epsilon \rightarrow 0$, like

$$
\sum C_{i}(\epsilon) p_{i}, \quad C_{i}=\sum_{j} C_{i j} \epsilon^{j},
$$

with the coefficient functions being functions (not necessarily finite at $\epsilon \rightarrow 0$ ) with purely rational coefficients $C_{i j}$, will not contain even zetas in the limit of $\epsilon \rightarrow 0$ (while odd zetas $\left\{\zeta_{2 n+1} \mid n \geq 1\right\}$ are expected and indeed appear in general).

3. Let $F(\epsilon)$ be any renormalized (and, thus, finite in the limit of $\epsilon \rightarrow 0$ ) combination of any p-integrals. The sole source of possible even zetas in $F(0)$ is the appearance of zetas (not necessarily even) in the renormalization factors involved in carrying out the renormalization of $\mathrm{F}$.

The theorem is a direct consequence of the following observation: all explicit results for 4-loop master p-integrals do depend on only the following three combinations of zetas:

$$
\hat{\zeta}_{3}=\zeta_{3}+\frac{3 \epsilon}{2} \zeta_{4}-\frac{5 \epsilon^{3}}{2} \zeta_{6}, \hat{\zeta}_{5}=\zeta_{5}+\frac{5 \epsilon}{2} \zeta_{6} \quad \text { and } \quad \zeta_{7} .
$$

The third point of the theorem provides the reason behind the absence of even zetas in the Adler function at order $\alpha_{s}^{3}$ and in the coefficient function $C^{B j p}$ at order $\alpha_{s}^{4}$. Indeed, both quantities are

(i) a finite combination of four-loop p-integrals;

(ii) the corresponding renormalizations require only the charge coupling renormalization at maximum three-loop order which is free from any zetas.

The fact that the Adler function at order $\alpha_{s}^{4}$ is also free from even zetas is not explained by the theorem. The reason is that in this order the Adler function is directly expressible in terms of five-loop p-integrals. The fact could be understood if the five-loop master p-integrals would obey a property similar to (5.1).

The hypothesis (suggested in [25]) is supported by another 5-loop physical quantity related to the scalar correlator. The corresponding Adler function is defined as follows:

$$
\begin{aligned}
& \widetilde{D}\left(Q^{2}\right)=\frac{Q^{2}}{6} \frac{\mathrm{d}}{\mathrm{d} Q^{2}} \frac{\widetilde{\Pi}\left(Q^{2}\right)}{Q^{2}}=\int_{0}^{\infty} \frac{Q^{2} \widetilde{R}(s) d s}{\left(s+Q^{2}\right)^{2}}, \\
& \widetilde{D}\left(Q^{2}\right)=1+\sum_{i=1}^{\infty} \widetilde{d}_{i}\left(\ell_{Q}\right) a_{s}^{i}(\mu),
\end{aligned}
$$


where $\mu$ is the $\overline{\mathrm{MS}}$ renormalization scale and $\ell_{Q}=\log \left(Q^{2} / \mu^{2}\right)$. The available results for $\tilde{d}_{i}$ read [50] (note that in order to save some space we set the number of quark flavours $n_{f}=3$ everywhere in this section):

$$
\begin{aligned}
\tilde{d}_{1}= & \frac{17}{3}-2 \ell_{Q}, \tilde{d}_{2}=\frac{9631}{144}-\frac{35}{2} \zeta_{3}-\frac{95}{3} \ell_{Q}+\frac{17}{4} \ell_{Q}^{2}, \\
\tilde{d}_{3}= & \frac{4748953}{5184}-\frac{91519}{216} \zeta_{3}-\frac{5}{2} \zeta_{4}+\frac{715}{12} \zeta_{5} \\
& +\ell_{Q}\left[-\frac{4781}{9}+\frac{475}{4} \zeta_{3}\right]+\frac{229}{2} \ell_{Q}^{2}-\frac{221}{24} \ell_{Q}^{3}, \\
\tilde{d}_{4}= & \frac{7055935615}{497664}-\frac{46217501}{5184} \zeta_{3}+\frac{192155}{216} \zeta_{3}^{2}-\frac{17455}{576} \zeta_{4} \\
& +\frac{455725}{432} \zeta_{5}-\frac{625}{48} \zeta_{6}-\frac{52255}{256} \zeta_{7} \\
& +\ell_{Q}\left[-\frac{97804997}{10368}+\frac{1166815}{288} \zeta_{3}+5 \zeta_{4}-\frac{24025}{48} \zeta_{5}\right] \\
& +\ell_{Q}^{2}\left[\frac{3008729}{1152}-\frac{16785}{32} \zeta_{3}\right]+\ell_{Q}^{3}\left[-\frac{51269}{144}\right]+\ell_{Q}^{4}\left[\frac{7735}{384}\right] .
\end{aligned}
$$

Unlike the vector case the scalar Adler function is full of even zetas starting already from three loops (the coefficient $\tilde{d}_{3}$ ). This is a natural consequence of the two facts

(i) the quantity by itself is not scale invariant but meets the following evolution equation

$$
\mu^{2} \frac{\mathrm{d}}{\mathrm{d} \mu^{2}} \widetilde{D}\left(\mu^{2} / Q^{2}, \alpha_{s}(\mu)\right)=-2 \gamma_{m}
$$

or, equivalently,

$$
\mu^{2} \frac{\partial}{\partial \mu^{2}} \widetilde{D}\left(\mu^{2} / Q^{2}, \alpha_{s}(\mu)\right)=-\left(2 \gamma_{m}+\beta\left(a_{s}\right) \frac{\partial}{\partial a_{s}}\right) \widetilde{D} .
$$

(ii) the quark mass anomalous dimension starts to depend on $\zeta_{3}$ at 3 loops and on $\zeta_{4}$ at 4 loops.

Let us now consider a related quantity, namely,

$$
\tilde{S}\left(\mu^{2} / Q^{2}, \alpha_{s}(\mu)\right) \equiv Q^{2} \frac{\partial}{\partial Q^{2}} \ln \widetilde{D},
$$

which, obviously, is scale-invariant and meets the evolution equation:

$$
\mu^{2} \frac{\mathrm{d}}{\mathrm{d} \mu^{2}} \tilde{S}\left(\mu^{2} / Q^{2}, \alpha_{s}(\mu)\right)=0 .
$$

Direct calculation gives:

$$
\begin{aligned}
\tilde{S}= & \sum_{i=1, \infty} \tilde{s}_{i}\left(\ell_{Q}\right) a_{s}^{i}(\mu), \\
\tilde{s}_{1}= & -2, \quad \tilde{s}_{2}=-\frac{61}{3}+\frac{9}{2} \ell_{Q}, \quad \tilde{s}_{3}=-\frac{20321}{72}+\frac{335}{4} \zeta_{3}+\ell_{Q}\left[\frac{199}{2}\right]-\frac{81}{8} \ell_{Q}^{2}, \\
\tilde{s}_{4}= & -\frac{48127465}{10368}+\frac{2050813}{864} \zeta_{3}-\frac{18305}{48} \zeta_{5} \\
& +\ell_{Q}\left[\frac{400873}{192}-\frac{9045}{16} \zeta_{3}\right]-\frac{5661}{16} \ell_{Q}^{2}+\frac{729}{32} \ell_{Q}^{3} .
\end{aligned}
$$


We observe that within the physical quantity $\tilde{S}$ all even zetas abounding in $\widetilde{D}$ neatly cancel each other not only in orders $a_{s}^{2}, a_{s}^{3}$ (as guaranteed by the theorem) but in order $\alpha_{s}^{4}$, too.

In fact, the absence of the even zetas in $\tilde{S}$ at order $a_{s}^{4}$ was discovered in [6] ten years before the result for $\tilde{d}_{4}$ became available. Indeed, as $\tilde{S}$ depends on $\mu$ and $Q^{2}$ only via the combination $\mu^{2} / Q^{2}$, the evolution equation (5.6) allows to construct the derivative $\frac{\partial}{\partial Q^{2}} \widetilde{D}$ at $(L+1)$-loop level merely from the knowledge of $\widetilde{D}$ at $L$ loops, the $\gamma_{m}$ and the $\beta$-function at $(L+1)$ loops.

As a result the expression (5.11) for $\tilde{S}$ was constructed ${ }^{7}$ by the authors of [6] from $\widetilde{D}$ at three loops and the quark mass anomalous dimension at four loops (the latter was the main result of their publication). In fact, the absence of $\zeta_{4}$ in $\tilde{S}$ was considered there as an extra cross-check for the both calculations of $\gamma_{m}$ and $\tilde{D}$.

Now, with the 5-loop $\gamma_{m}$ at hands we can easily construct the function $\tilde{S}$ at six-loop level (that is to order $\alpha_{s}^{5}$ !). The result for $\tilde{s}_{5}$ reads

$$
\begin{aligned}
\tilde{s}_{5}= & -\frac{43177218695}{497664}+\frac{3589509737}{62208} \zeta_{3}-\frac{20395}{3} \zeta_{3}^{2}-\frac{1335}{128} \zeta_{4}-\frac{200406415}{31104} \zeta_{5}+\frac{3285415}{2304} \zeta_{7} \\
& +\ell_{Q}\left[\frac{105141365}{2304}-\frac{2144623}{96} \zeta_{3}+\frac{54915}{16} \zeta_{5}\right]+\ell_{Q}^{2}\left[-\frac{2569659}{256}+\frac{81405}{32} \zeta_{3}\right] \\
& +\ell_{Q}^{3}\left[\frac{17631}{16}\right]+\ell_{Q}^{4}\left[-\frac{6561}{128}\right]
\end{aligned}
$$

and it indeed contains $\zeta_{4}$.

\section{Conclusions}

We have presented the result for the five-loop quark mass anomalous dimension $\gamma_{m}$ for the case of a generic gauge group as well as explicit formulas which we have used. We have demonstrated that at the level $\alpha_{s}^{5}$ the even zetas do appear in physical observables expressible in terms of massless propagators.

\section{Acknowledgments}

The work by K.G. Chetykin and J.H. Kühn was supported by the Deutsche Forschungsgemeinschaft through CH1479/1-1. The work of P.A. Baikov is supported in part by the grant NSh-7989.2016.2 of the President of Russian Federation and by the grant RFBR 17-02-00175A of the Russian Foundation for Basic Research.

Note added. The calculation of $\gamma_{m}$ presented here was about its completion when we were informed about the existence of a result [41] (not then yet submitted to the Archive) for the same quantity. After a few days we have got our result (4.2) which happens to be in full agreement with that from [41]. We thank the authors of [41] for informing us on their results.

\footnotetext{
${ }^{7}$ To be honest, the authors of this work were dealing not with the function $\tilde{S}$ but with a closely related one; the difference is not essential for our reasonings.
} 
Open Access. This article is distributed under the terms of the Creative Commons Attribution License (CC-BY 4.0), which permits any use, distribution and reproduction in any medium, provided the original author(s) and source are credited.

\section{References}

[1] P.A. Baikov, K.G. Chetyrkin and J.H. Kühn, Quark Mass and Field Anomalous Dimensions to $\mathcal{O}\left(\alpha_{s}^{5}\right)$, JHEP 10 (2014) 076 [arXiv:1402.6611] [INSPIRE].

[2] R. Tarrach, The Pole Mass in Perturbative QCD, Nucl. Phys. B 183 (1981) 384 [InSPIRE].

[3] O. Tarasov, Anomalous Dimensions Of Quark Masses In Three Loop Approximation, JINR-P2-82-900 (1982) [INSPIRE].

[4] S.A. Larin, The Renormalization of the axial anomaly in dimensional regularization, Phys. Lett. B 303 (1993) 113 [hep-ph/9302240] [INSPIRE].

[5] K.G. Chetyrkin, Quark mass anomalous dimension to $O\left(\alpha_{s}^{4}\right)$, Phys. Lett. B 404 (1997) 161 [hep-ph/9703278] [INSPIRE].

[6] J.A.M. Vermaseren, S.A. Larin and T. van Ritbergen, The four loop quark mass anomalous dimension and the invariant quark mass, Phys. Lett. B 405 (1997) 327 [hep-ph/9703284] [INSPIRE].

[7] P.A. Baikov, K.G. Chetyrkin and J.H. Kühn, Towards QCD running in 5 loops: quark mass anomalous dimension, PoS (RADCOR 2013) 056 [arXiv: 1402.6606] [INSPIRE].

[8] P.A. Baikov, K.G. Chetyrkin and J.H. Kühn, Five-Loop Running of the QCD coupling constant, Phys. Rev. Lett. 118 (2017) 082002 [arXiv:1606. 08659] [INSPIRE].

[9] P.A. Baikov, K.G. Chetyrkin and J.H. Kühn, The $\beta$-function of Quantum Chromodynamics and the effective Higgs-gluon-gluon coupling in five-loop order, PoS (LL2016) 010 [INSPIRE].

[10] K. Chetyrkin and J.H. Kühn, Precision Measurements in Electron-Positron Annihilation: Theory and Experiment, PoS (LL2016) 047 [arXiv:1607.08374] [INSPIRE].

[11] G. 't Hooft and M.J.G. Veltman, Regularization and Renormalization of Gauge Fields, Nucl. Phys. B 44 (1972) 189 [INSPIRE].

[12] W.A. Bardeen, A.J. Buras, D.W. Duke and T. Muta, Deep Inelastic Scattering Beyond the Leading Order in Asymptotically Free Gauge Theories, Phys. Rev. D 18 (1978) 3998 [INSPIRE].

[13] K.G. Chetyrkin, J.H. Kühn and A. Kwiatkowski, $Q C D$ corrections to the $e^{+} e^{-}$cross-section and the $Z$ boson decay rate, hep-ph/9503396 [INSPIRE].

[14] A.A. Vladimirov, Method for Computing Renormalization Group Functions in Dimensional Renormalization Scheme, Theor. Math. Phys. 43 (1980) 417 [InSPIRE].

[15] D.I. Kazakov, O.V. Tarasov and A.A. Vladimirov, Calculation of Critical Exponents by Quantum Field Theory Methods, Sov. Phys. JETP 50 (1979) 521 [inSPIRE].

[16] K.G. Chetyrkin, A.L. Kataev and F.V. Tkachov, New Approach to Evaluation of Multiloop Feynman Integrals: The Gegenbauer Polynomial x Space Technique, Nucl. Phys. B 174 (1980) 345 [INSPIRE].

[17] O.V. Tarasov, A.A. Vladimirov and A.Yu. Zharkov, The Gell-Mann-Low Function of QCD in the Three Loop Approximation, Phys. Lett. B 93 (1980) 429 [INSPIRE]. 
[18] K.G. Chetyrkin and V.A. Smirnov, $R^{*}$ operation corrected, Phys. Lett. B 144 (1984) 419 [INSPIRE].

[19] K.G. Chetyrkin, Corrections of order $\alpha_{s}^{3}$ to $R_{\text {had }}$ in $p Q C D$ with light gluinos, Phys. Lett. B 391 (1997) 402 [hep-ph/9608480] [INSPIRE].

[20] N.N. Bogoliubov and O.S. Parasiuk, On the Multiplication of the causal function in the quantum theory of fields, Acta Math. 97 (1957) 227.

[21] N. Bogoliubov and D. Shirkov, Introduction to the Theory of Quantized Fields, 3rd edition, John Wiley \& Sons Inc. (1980).

[22] K.G. Chetyrkin, Combinatorics of $R-, R^{-1}$ - and $R^{*}$-operations and asymptotic expansions of Feynman integrals in the limit of large momenta and masses, arXiv:1701.08627 [INSPIRE].

[23] D.V. Batkovich and M. Kompaniets, Toolbox for multiloop Feynman diagrams calculations using $R^{*}$ operation, J. Phys. Conf. Ser. 608 (2015) 012068 [arXiv:1411.2618] [INSPIRE].

[24] P. Nogueira, Automatic Feynman graph generation, J. Comput. Phys. 105 (1993) 279 [INSPIRE].

[25] P.A. Baikov and K.G. Chetyrkin, Four Loop Massless Propagators: An Algebraic Evaluation of All Master Integrals, Nucl. Phys. B 837 (2010) 186 [arXiv:1004.1153] [INSPIRE].

[26] R.N. Lee, A.V. Smirnov and V.A. Smirnov, Master Integrals for Four-Loop Massless Propagators up to Transcendentality Weight Twelve, Nucl. Phys. B 856 (2012) 95 [arXiv: 1108.0732] [INSPIRE].

[27] A.V. Smirnov and M. Tentyukov, Four Loop Massless Propagators: a Numerical Evaluation of All Master Integrals, Nucl. Phys. B 837 (2010) 40 [arXiv:1004.1149] [INSPIRE].

[28] R.N. Lee, Presenting LiteRed: a tool for the Loop InTEgrals REDuction, arXiv:1212.2685 [INSPIRE].

[29] R.N. Lee, LiteRed 1.4: a powerful tool for reduction of multiloop integrals, J. Phys. Conf. Ser. 523 (2014) 012059 [arXiv: 1310.1145] [INSPIRE].

[30] T. Ueda, B. Ruijl and J.A.M. Vermaseren, Forcer: a FORM program for 4-loop massless propagators, PoS (LL2016) 070 [arXiv: 1607.07318] [INSPIRE].

[31] F. Herzog, B. Ruijl, T. Ueda, J.A.M. Vermaseren and A. Vogt, FORM, Diagrams and Topologies, PoS (LL2016) 073 [arXiv: 1608.01834] [INSPIRE].

[32] P.A. Baikov, A practical criterion of irreducibility of multi-loop Feynman integrals, Phys. Lett. B 634 (2006) 325 [hep-ph/0507053] [INSPIRE].

[33] P.A. Baikov, Explicit solutions of the three loop vacuum integral recurrence relations, Phys. Lett. B 385 (1996) 404 [hep-ph/9603267] [INSPIRE].

[34] J.A.M. Vermaseren, New features of FORM, math-ph/0010025 [INSPIRE].

[35] M. Steinhauser, T. Ueda and J.A.M. Vermaseren, Parallel versions of FORM and more, Nucl. Part. Phys. Proc. 261-262 (2015) 45 [arXiv:1501.07119] [INSPIRE].

[36] W.E. Caswell and A.D. Kennedy, A simple approach to renormalization theory, Phys. Rev. D 25 (1982) 392 [INSPIRE].

[37] J.C. Collins, Normal Products in Dimensional Regularization, Nucl. Phys. B 92 (1975) 477 [INSPIRE]. 
[38] M. Misiak and M. Münz, Two loop mixing of dimension five flavor changing operators, Phys. Lett. B 344 (1995) 308 [hep-ph/9409454] [INSPIRE].

[39] T. van Ritbergen, J.A.M. Vermaseren and S.A. Larin, The Four loop $\beta$-function in quantum chromodynamics, Phys. Lett. B 400 (1997) 379 [hep-ph/9701390] [INSPIRE].

[40] K.G. Chetyrkin, M. Misiak and M. Münz, $\beta$-functions and anomalous dimensions up to three loops, Nucl. Phys. B 518 (1998) 473 [hep-ph/9711266] [INSPIRE].

[41] T. Luthe, A. Maier, P. Marquard and Y. Schröder, Five-loop quark mass and field anomalous dimensions for a general gauge group, JHEP 01 (2017) 081 [arXiv:1612.05512] [INSPIRE].

[42] T. Luthe, A. Maier, P. Marquard and Y. Schröder, Complete renormalization of QCD at five loops, JHEP 03 (2017) 020 [arXiv: 1701.07068] [INSPIRE].

[43] A.A. Vladimirov, Methods of Multiloop Calculations and the Renormalization Group Analysis of $\phi^{4}$ Theory, Theor. Math. Phys. 36 (1979) 732 [InSPIRE].

[44] K.G. Chetyrkin, A.L. Kataev and F.V. Tkachov, Higher Order Corrections to $\sigma_{\text {tot }}\left(e^{+} e^{-} \rightarrow\right.$ Hadrons) in Quantum Chromodynamics, Phys. Lett. B 85 (1979) 277 [InSPIRE].

[45] S.G. Gorishnii, A.L. Kataev and S.A. Larin, The $O\left(\alpha_{s}^{3}\right)$-corrections to $\sigma_{\text {tot }}\left(e^{+} e^{-} \rightarrow\right.$ hadrons $)$ and $\Gamma\left(\tau^{-} \rightarrow \nu_{\tau}+\right.$ hadrons) in QCD, Phys. Lett. B 259 (1991) 144 [INSPIRE].

[46] F. Herzog, B. Ruijl, T. Ueda, J.A.M. Vermaseren and A. Vogt, The five-loop $\beta$-function of Yang-Mills theory with fermions, JHEP 02 (2017) 090 [arXiv: 1701.01404] [INSPIRE].

[47] T. van Ritbergen, A.N. Schellekens and J.A.M. Vermaseren, Group theory factors for Feynman diagrams, Int. J. Mod. Phys. A 14 (1999) 41 [hep-ph/9802376] [INSPIRE].

[48] P.A. Baikov, K.G. Chetyrkin and J.H. Kühn, Adler Function, Bjorken Sum Rule and the Crewther Relation to Order $\alpha_{s}^{4}$ in a General Gauge Theory, Phys. Rev. Lett. 104 (2010) 132004 [arXiv: 1001.3606] [INSPIRE].

[49] D.J. Broadhurst, Dimensionally continued multiloop gauge theory, hep-th/9909185 [INSPIRE].

[50] P.A. Baikov, K.G. Chetyrkin and J.H. Kühn, Scalar correlator at $\mathcal{O}\left(\alpha_{s}^{4}\right)$, Higgs decay into b-quarks and bounds on the light quark masses, Phys. Rev. Lett. 96 (2006) 012003 [hep-ph/0511063] [INSPIRE]. 